\title{
Probing the inner halo and IVC gas through the Local Interstellar Chimney
}

\author{
B. Y. Welsh ${ }^{1,4}$, S. Sallmen ${ }^{2}$, and R. Lallement ${ }^{3}$ \\ ${ }^{1}$ Experimental Astrophysics Group, Space Sciences Laboratory, UC Berkeley, Berkeley, CA 94720, USA \\ 2 Department of Physics, Univ. of Wisconsin-La Crosse, La Crosse, WI 54601, USA \\ 3 Service d'Aéronomie du CNRS, 91371 Verrières-le-Buisson, France \\ ${ }^{4}$ Eureka Scientific Inc., 2452 Delmer St, Oakland, CA 94602, USA
}

Received 19 September 2003 / Accepted 9 October 2003

\begin{abstract}
We present an absorption study of the interstellar gas at high positive galactic latitudes in the direction close to the axis of the Local Chimney (LC), which is an extension of the rarefied local cavity that reaches out from the galactic disk to a $z$-distance of at least $250 \mathrm{pc}$ into the lower halo region. Our study includes high-resolution $\left(R \sim 1.7 \mathrm{~km} \mathrm{~s}^{-1}\right)$ spectral observations of the interstellar NaI and CaII absorption lines seen towards 6 early-type stars with distances ranging from 225 to $500 \mathrm{pc}$ contained within a radius of $\sim 6^{\circ}$ along the sight-line $\left(l=160^{\circ}, b=+55^{\circ}\right)$. These visible data are supplemented with far-ultraviolet absorption measurements of the interstellar sight-lines towards two hot white dwarf stars, RE J1043+490 $(d=230 \mathrm{pc})$ and RE J1059+512 $(d=315 \mathrm{pc})$, taken with the NASA Far Ultraviolet Spectroscopic Explorer (FUSE) satellite. Our observations reveal interstellar gas clouds with velocities in the -20 to $-60 \mathrm{~km} \mathrm{~s}^{-1}$ range that appear to be falling towards the galactic disk. In particular, we have detected absorption with a velocity of $V_{\text {helio }} \sim-55 \mathrm{~km} \mathrm{~s}^{-1}$ towards two sight-lines (HD 89501 and HD 88545) that can be associated with an intermediate velocity (IV) cloud called the IV Arch. Our observations place a probable $z$-distance to this IVC of $275-320 \mathrm{pc}$, this being much nearer than previously thought.

The far UV spectra of the two hot white dwarfs show only a few $(\sim 10)$ interstellar absorption lines, which is indicative of the very low density phase of the interstellar gas contained within the LC region. We have derived relative elemental abundance ratios for $\mathrm{C}, \mathrm{N}, \mathrm{O}, \mathrm{Si}, \mathrm{Ar}$ and $\mathrm{Fe}$ for these two sight-lines and find that the abundance patterns are very similar to those found for other sight-lines in the local cavity. The high ionization line of OVI $\lambda 1032 \AA$ has been detected towards RE J1043+490 with a column density of $7 \times 10^{12} \mathrm{~cm}^{-2}$, which is consistent with the average space density of this ion recently found in the Local Bubble region.

Finally, we (tentatively) propose a possible connection between the formation of the Gould Belt, the Local Bubble cavity, the Local Chimney and the overlying IV Arch clouds.
\end{abstract}

Key words. ISM: atoms - ISM: bubbles - Galaxy: solar neighborhood

\section{Introduction}

The general physical and dynamical characteristics of the neutral and ionized gas of the interstellar medium (ISM) found throughout both the galactic disk and galactic halo are now fairly well-understood (Dyson \& Williams 1997). This interstellar gas exists in four distinct phases: the cold neutral medium (CNM: $T \sim 100 \mathrm{~K}$ ), the warm neutral medium (WNM: $T \sim 6000 \mathrm{~K}$ ), the warm ionized medium (WIM: $T \sim$ $8000 \mathrm{~K}$ ) and the hot and ionized medium (HIM: $T \sim 10^{6} \mathrm{~K}$ ). Over the past decade we have been studying the neutral interstellar gas residing within the solar neighborhood (i.e. $<300 \mathrm{pc}$ ) in order to accurately determine both its spatial distribution and its physical and kinematical state (Sfeir et al. 1999; Lallement et al. 2003). This local region of the Galaxy contains a large interstellar cavity (diameter $\sim 150 \mathrm{pc}$ ) of extremely low neutral gas density $\left(n_{\mathrm{H}}=0.005 \mathrm{~cm}^{-3}\right)$ often termed the "Local
Bubble" or "local cavity" (Frisch 1995). Although there is still much debate as to the temperature and pressure of the ionized gas contained within this rarefied cavity (Jenkins 2002), we do know that this region is surrounded in many directions by an absorption boundary (or "wall") of cold and relatively dense $\left(N(\mathrm{HI})>5 \times 10^{19} \mathrm{~cm}^{-2}\right.$ ) neutral gas (Sfeir et al. 1999). The most accurate 3D galactic contours of this absorption boundary to the local cavity have recently been determined by Lallement et al. (2003) who have sampled over 1000 interstellar sightlines within $350 \mathrm{pc}$ of the Sun using high-resolution absorption measurements of the NaI D-lines. These data clearly show that the local cavity possesses a well defined neutral boundary in the galactic plane, but at high galactic latitudes it appears open-ended in both galactic hemispheres with no welldetermined dense (neutral) absorption boundary to a $z$-height of at least $250 \mathrm{pc}$. This $25^{\circ}$ diameter tube-like extension, called the "Local Interstellar Chimney" (LC), points in the direction 
towards $\left(l=155^{\circ}, b=+58^{\circ}\right)$ in the northern hemisphere and towards $\left(l=330^{\circ}\right)$ in the southern hemisphere, and was first revealed in mapping the galactic distribution of EUV sources by Welsh et al. (1999). Typically, the neutral hydrogen column density measured towards targets lying within the confines of the LC is $N(\mathrm{HI})<2 \times 10^{19} \mathrm{~cm}^{-2}$ and the cavity tube is oriented with respect to the Galaxy in a particularly interesting direction. Its axis lies almost perpendicular to that of the Gould Belt association of early-type stars, suggesting that this inclination is consistent with the LC being squeezed by the expansion of the supershells surrounding the local cavity. In addition, because of its low neutral hydrogen density (and associated low infrared cirrus structure) many of the "deep fields" currently being surveyed in astronomy are located in sight-lines within \pm 15 degrees of the LC axis.

The presence of the low density LC within our solar neighborhood provides us with the unique prospect of being able to sample the interaction region between the outer region of the thick galactic disk and the inner region of the galactic halo without the complication of intervening, potentially obscuring interstellar absorption structures. We note that the transition region between the galactic disk and the inner halo $(z \sim 300-600 \mathrm{pc})$ is still poorly understood. Dickey et al. (1981) have shown that the $z$-distribution of cold $(T<500 \mathrm{~K})$ $\mathrm{HI}$ absorption is distributed roughly as a Gaussian with a scale height of $\sim 150 \mathrm{pc}$, whereas the total gas distribution shown by the emission includes another component, a broad exponential ("Lockman Layer") with a scale height of $\sim 500$ pc which consists almost exclusively of warm $(T \sim 6000 \mathrm{~K})$ and diffuse gas. The term "layer" (Lockman 1984) is somewhat misleading since high-resolution absorption studies have shown this "uniform layer" to be in the form of many discrete (neutral and partially ionized) clouds with distances up to $1 \mathrm{kpc}$ (Albert et al. 1991). Observations along several halo sight-lines show no kinematic evidence for a physical separation between the ionized and neutral material (Howk \& Savage 1999), which is consistent with a partially ionized medium in which the neutrals and ions are well mixed, with the latter being surrounded by ionized cloud envelopes (McKee \& Ostriker 1977). The likely source of ionization of the WIM gas in the halo is photoionization by OB stars, although other mechanisms such as supernovae and cosmic ray interaction may be non-negligible contributors to the total ionization field (Reynolds 1984).

Recently, we have sampled the ISM gas along the southern extent of the LC (i.e. at highly negative galactic latitudes) using high-resolution $\left(R \sim 1 \mathrm{~km} \mathrm{~s}^{-1}\right)$ absorption measurements of the interstellar NaI and CaII K-lines observed towards 7 earlytype stars with distances up to $z \sim 800 \mathrm{pc}$ (Crawford et al. 2002). These observations have revealed a pattern of gaseous absorption in the inner-halo interaction region that is inconsistent with the well-accepted picture previously forwarded by Lockman and others. Our observations failed to detect the gas of the WNM ( $T \sim 6000 \mathrm{~K}$ ) normally thought to be associated with the Lockman Layer and instead revealed a population of colder $(T<2000 \mathrm{~K})$, diffuse cloudlets with physical properties similar to the gas found in the local ISM (i.e. relatively cool interiors surrounded by warmer and/or more turbulent haloes). These lower halo cloudlets were found to be mostly falling towards the galactic disk with velocities of $\sim-20 \mathrm{~km} \mathrm{~s}^{-1}$, which is consistent with the galactic fountain model of Shapiro \& Field (1976) in which hot, ionized gas rises into the halo as a fountain and descends onto the disk in the form of neutral clouds. We note that since the publication of the Crawford et al. results, Lockman (2002) has reported the discovery of a (ubiquitous) population of HI clouds lying between $500 \mathrm{pc}$ and $1 \mathrm{kpc}$ below the galactic plane with hydrogen column densities of $N(\mathrm{HI}) \sim 2 \times 10^{19} \mathrm{~cm}^{-2}$, temperatures of $<2000 \mathrm{~K}$ and masses of $\sim 50 M_{\odot}$. It seems highly probable that this is the type of halo cloudlet that has been detected by the $\mathrm{NaI}$ and CaII observations of Crawford et al. (2002).

Maiz-Apellaniz (2001) has argued that supernovae, produced by stars in the Lower Centaurus Crux OB association may have created the local cavity some $6 \mathrm{Myr}$ ago. If this scenario is true, then the LC could be a conduit for any expanding hot and ionized gas from the initial explosion that has broken out of the galactic disk and entered into the lower halo. Welsh et al. (2002) have used the NASA FUSE satellite to sample the interstellar gas found within the inner region of the northern extension of the LC, but were unsuccessful in detecting absorption due to the OVI ion $(T \sim 300000 \mathrm{~K})$ towards the hot white dwarf star RE J1032+532 situated in the direction $\left(l=157.5^{\circ}, b=+53.2^{\circ}\right)$ at a distance $116 \mathrm{pc}$ along the LC. However, the OVI ion was detected in emission at two positions very close to this sight-line, indicating that hot and highly ionized gas is present (at some distance $>116 \mathrm{pc}$ ) along the projection of the LC. Although the velocities of both of the observed OVI emission lines were not well constrained (at $V_{\text {helio }}=-20 \pm 22 \mathrm{~km} \mathrm{~s}^{-1}$ and $-55 \pm 30 \mathrm{~km} \mathrm{~s}^{-1}$ ), the data are consistent with the notion that the hot emitting gas may be falling towards the galactic disk. However, just how this hot phase of the ISM is coupled to the colder, diffuse clouds within the local cavity and Local Chimney structures, and what are the kinematic and physical characteristics of the gas clouds in the disk-inner halo region at high positive galactic latitudes still remain largely unknown.

In this Paper we sample the neutral gas lying in the outer disk-inner halo region using high spectral resolution $(R \sim$ $1.7 \mathrm{~km} \mathrm{~s}^{-1}$ ) measurements of the $\mathrm{NaI}$ and CaII interstellar lines seen in absorption towards 6 early-type stars that lie within the northern galactic extension of the LC out to a $z$-distance of $\sim 380 \mathrm{pc}$. In addition, we also present far UV absorption measurements of interstellar gas in two sight-lines towards the hot white dwarf stars RE J1043+490 $(d=230 \mathrm{pc})$ and RE J1059+512 $(d=315$ pc), both of which lie within the boundary of the LC. This region of the sky contains the famous Lockman Window $\left(l=149^{\circ}, b=+54^{\circ}\right)$, which has the lowest HI column density of any section of the northern sky (Lockman et al. 1986). Our sight-lines are also contained within a region mapped by Hausen et al. (2002), in which unusually weak levels of interstellar $\mathrm{H}-\alpha$ emission were found. Thus, the presently sampled sight-lines are all contained within a truly low column density window through the galactic disk, which is depleted both in HI and HII gas. Our observations reveal the presence of mostly negative velocity (neutral) gas clouds that appear to be falling towards the galactic disk from the lower halo. We provide compelling evidence that the gas observed with a velocity 
Table 1. stellar target information.

\begin{tabular}{ccccc}
\hline \hline Star & $(l, b)$ & $m_{v}$ & Sp & $\begin{array}{c}\text { Hipparcos distance } \\
(\mathrm{pc})\end{array}$ \\
\hline HD 86274 & $\left(166.0^{\circ},+50.0^{\circ}\right)$ & 6.7 & B9IV & $290(+90,-55)$ \\
HD 87972 & $\left(167.3^{\circ},+52.2^{\circ}\right)$ & 8.6 & A3 & $348(+280,-100)$ \\
HD 88545 & $\left(155.9^{\circ},+49.7^{\circ}\right)$ & 8.1 & A0 & $500(+425,-155)$ \\
HD 89501 & $\left(156.0^{\circ},+50.8^{\circ}\right)$ & 8.7 & A2 & $412(+310,-125)$ \\
HD 91311 & $\left(157.3^{\circ},+53.4^{\circ}\right)$ & 6.5 & A1V & $225(+45,-30)$ \\
HD 95126 & $\left(165.4^{\circ},+61.1^{\circ}\right)$ & 8.9 & A0V & $435 \pm 80 *$ \\
RE J1043+490 & $\left(162.7^{\circ},+57.0^{\circ}\right)$ & 16.1 & DA & $230 \pm 45 * *$ \\
RE J1059+512 & $\left(156.4^{\circ},+57.8^{\circ}\right)$ & 16.8 & DA & $315 \pm 60 * *$ \\
\hline$*$ * distance from absolute and visual magnitudes, ** = distance from Vennes et al. $(1997)$ \\
\hline
\end{tabular}

of $V_{\text {helio }} \sim-55 \mathrm{~km} \mathrm{~s}^{-1}$ and a $z$-height $>275 \mathrm{pc}$ is associated with the Intermediate Velocity Arch feature in the lower halo. We also propose that there could be a link between the direction of the opening of the LC into the inner halo and the enhancement in the number of Intermediate Velocity Clouds (IVCs) observed at high latitude in the northern galactic hemisphere.

\section{Observations and data reduction}

In Table 1 we list the 8 stars we have observed, together with their galactic positions, visual magnitudes, spectral types and distance estimates. Accurate Hipparcos satellite distances are available for 5 of the stars listed in Table 1 (ESA 1997). Distances to the two hot white dwarf stars are taken from Vennes et al. (1997) and are accurate to $\sim \pm 20 \%$. We have estimated a stellar photometric distance to HD 95126 of $435 \pm$ $80 \mathrm{pc}$ using the absolute-visual magnitude relationship under the assumption of zero redddening.

Interstellar absorption data for the $\mathrm{NaI}$ and CaII K-lines were obtained for 6 of the targets during the nights of Dec. 30th 2002-Jan. 1st 2003 using the coude echelle (cs21-e1) spectrograph at the $2.7 \mathrm{~m}$ telescope of the McDonald Observatory, Texas (Tull et al. 1995). These data were recorded using the TK3 Tektronix $2048 \times 2048$ CCD detector at a spectral resolving power of $\sim 180000\left(1.7 \mathrm{~km} \mathrm{~s}^{-1}\right)$. Typically, total exposure times of $60 \mathrm{~min}$ were required to obtain a $\mathrm{S} / \mathrm{N}$ ratio of $\sim 15: 1$ at $\mathrm{NaI}(5890 \AA$ ), whereas an exposure of 2 hours was required to produce a similar $\mathrm{S} / \mathrm{N}$ at CaII (3933 $\AA$ ).

The visible spectral data were then extracted and reduced in a manner similar to that described in Sfeir et al. (1999) which consists of background and detector bias subtraction, cosmic ray removal, flatfielding, optimal spectral order extraction and wavelength assignment from a third-order polynomial fit to the Th-Ar calibration spectra. The absolute wavelength accuracy of the resultant calibrated visible data was $\sim \pm 0.02 \AA$ and all velocities reported in this Paper are in the heliocentric frame of reference. To convert from heliocentric to local standard of rest (lsr) velocities for these data, an average correction of $+3.5 \mathrm{~km} \mathrm{~s}^{-1}$ is required.

Finally, the levels of telluric water vapor absorption line contamination (which particularly affect the NaI D2-line region)) were removed using a computed synthetic atmospheric transmission spectrum described in Lallement et al. (1993).

In addition to these ground based visible data, we have obtained far-ultraviolet absorption observations (over the wavelength range $2912-1180 \AA$ ) of two hot white dwarf stars, RE J1043+490 and RE J1059+512, using the NASA FUSE satellite (Moos et al. 2000). These data were taken using the LWRS $(30 \times 30$ arcsec $)$ spectrograph aperture, with events recorded in the detector photon time-tag mode which resulted in a total observation time of $75000 \mathrm{~s}$ for RE J1043+490 and $35000 \mathrm{~s}$ for RE J1059+512. Data in each of the 8 spectral channels of the FUSE instrument were individually processed using versions CFv2.2.3 and CFv2.1.6 of the FUSE science data reduction (CALFUSE) pipeline, which correct for geometric image distortions, background subtraction, image thermal drifts, detector deadtime and wavelength calibration (Sahnow et al. 2000). Both of these white dwarf spectra were fairly well exposed for wavelengths $>1000 \AA$ with a typical S/N ratio of $\sim 15: 1$. However, the spectra at shorter wavelengths (particularly for RE J1059+512) were of an inferior S/N ratio $(<10: 1)$. The resultant spectral resolution of the far UV data was $R \sim 21000$ (i.e. $14 \mathrm{~km} \mathrm{~s}^{-1}$ ), as determined from the fitting of weak interstellar absorption lines in the spectra.

We searched the entire $\lambda 912-1180 \AA$ wavelength range for possible interstellar line detections in both of the white dwarf FUSE spectra, using the list of absorption lines detected towards 30 white dwarfs in the local ISM by Lehner et al. (2003) as a guide. Detections of these interstellar lines for each target were only deemed valid if the absorption line was present in more than one of the FUSE spectral channnels. Using this criterion, together with the proviso that the central absorption component of a line should have a similar (relative) wavelength shift from its vacuum wavelength as listed in Morton (1991), we have firmly detected 11 different UV interstellar lines towards the 2 hot white dwarfs. We note that these measured wavelength shifts (which are due to a combination of the actual velocity shift of the interstellar line from its rest wavelength plus an undetermined wavelength inaccuracy in the derived FUSE wavelength scale) are quite distinct from those values found for the far UV stellar lines of both stars in each of the FUSE spectral channels. Several of the stronger interstellar lines recorded towards both RE J1043+490 and RE J1059+512 contain at least one extra absorption component at a velocity separation of $\sim-35 \mathrm{~km} \mathrm{~s}^{-1}$ from the central absorbing component, which also helped in confirming their origin as interstellar and not stellar. Thus in total, including the visible $\mathrm{NaI}$ and CaII lines, we are able to present data for 13 interstellar absorption lines detected within the LC sight-line. 
Table 2. NaI and CaII absorption line best-fit parameters.

\begin{tabular}{|c|c|c|c|c|c|c|c|}
\hline Star & $\begin{array}{c}V \\
\mathrm{~km} \mathrm{~s}^{-1} \\
\end{array}$ & $b$ & $\begin{array}{c}N \\
\left(10^{10} \mathrm{~cm}^{-2}\right) \\
\end{array}$ & & $\begin{array}{c}V \\
\mathrm{~km} \mathrm{~s}^{-1}\end{array}$ & $b$ & $\begin{array}{c}N \\
\left(10^{10} \mathrm{~cm}^{-2}\right)\end{array}$ \\
\hline HD 86274 & & & & & & & \\
\hline $\begin{array}{c}\text {...NaI... } \\
\text { HD } 87972\end{array}$ & & & $<2.0$ & ...CaII... & -20.0 & 1.8 & $31.0 \pm 3.0$ \\
\hline ...NaI... & $\begin{array}{l}-30.8 \\
-26.0 \\
-17.6\end{array}$ & $\begin{array}{l}4.3 \\
2.1 \\
2.5\end{array}$ & $\begin{array}{c}14.5 \pm 5.0 \\
44.0 \pm 10 \\
5.2 \pm 2.0\end{array}$ & ...CaII... & -18.4 & 2.4 & $33.8 \pm 7.0$ \\
\hline HD 88545 & & & & & & & \\
\hline ...NaI... & & & $<2.0$ & ...CaII... & $\begin{array}{l}-51.0 \\
-38.9 \\
-33.7 \\
-14.6\end{array}$ & $\begin{array}{l}2.6 \\
3.8 \\
1.7 \\
2.2\end{array}$ & $\begin{array}{c}12.7 \pm 3.0 \\
23.0 \pm 5.0 \\
18.9 \pm 4.0 \\
9.9 \pm 4.0\end{array}$ \\
\hline $\begin{array}{c}\text { HD } 89501 \\
\text {...NaI... }\end{array}$ & & & $<3.0$ & ...CaII... & $\begin{array}{l}-59.5 \\
-15.5\end{array}$ & $\begin{array}{l}1.3 \\
1.7\end{array}$ & $\begin{array}{c}41.5 \pm 10.0 \\
23.5 \pm 5\end{array}$ \\
\hline $\begin{array}{c}\text { HD } 91311 \\
\ldots \text { NaI... } \\
\text { HD } 95126\end{array}$ & & & $<1.5$ & ...CaII... & & & $<2.0$ \\
\hline ...NaI... & -56.9 & 2.0 & $13.0 \pm 3.0$ & ...CaII... & $\begin{array}{l}-62.0 \\
-56.0\end{array}$ & $\begin{array}{l}3.2 \\
0.9\end{array}$ & $\begin{array}{l}25.5 \pm 5.0 \\
10.8 \pm 3.5 \\
\end{array}$ \\
\hline
\end{tabular}

\section{Interstellar analysis}

We have determined the local stellar continua for all of the visible and UV interstellar lines using a multi-order polynomial in order to produce resultant residual intensity profiles. These profiles were then fit with one or more absorption components (identified with interstellar "clouds") using a line-fitting program described in Sfeir et al. (1999). This program assigns a 3-parameter theoretical fit to the observed profiles by assigning values for the interstellar gas cloud velocity, $V$, a Gaussian velocity dispersion, $b$ and a cloud component column density, $\mathrm{N}$. For the case of the NaI doublet, the best-fit was peformed simultaneously on both of the D1 and D2 line profiles. All of the visible and far UV profile fits were performed using the minimum number of absorption components. The addition of an extra absorption component was only deemed necessary if the chi-squared residual error between the observed and computed data points decreased by more than 11.1 (Vallerga et al. 1993).

Since absolute values to the FUSE wavelength scale are not known to within $\sim \pm 20 \mathrm{~km} \mathrm{~s}^{-1}$ (Sahnow et al. 2000), we have assumed that the deepest (largest column density) absorption component of all the far UV interstellar lines detected towards both hot white dwarfs are formed at $V_{\text {helio }}=0 \mathrm{~km} \mathrm{~s}^{-1}$. This assumption is based on the HST spectral observations by Holberg et al. (1999) of RE J1032+532, a hot white dwarf star that is located in a nearby sight-line along the LC at a distance of $110 \mathrm{pc}$. The shifts applied to the FUSE data to achieve this were small, being $+12 \mathrm{~km} \mathrm{~s}^{-1}$ for RE J1042+490 and $+5 \mathrm{~km} \mathrm{~s}^{-1}$ for RE J1059+512.

The best-fit values of $V, b$ and $N$ for the observed $\mathrm{NaI}$ and CaII lines towards the 6 targets are listed in Table 2, and the model fits are shown superposed over their respective residual intensity profiles in Figs. 1 and 2. The best-fit data for the far-ultraviolet interstellar lines observed towards the two white dwarf stars are listed in Table 3, and their respective residual intensity profiles and associated model fits are shown in Figs. 3 and 4. For the interstellar lines that were not detected in the spectra we have listed conservative $(3-\sigma)$ upper limits to their column densities, based on measurement of the equivalent width of the largest noise feature near the expected wavelength of the interstellar absorption line. In Fig. 1 we show the actual spectral data around the D2 line's wavelength that was used to determine some of these upper limits.

\section{Discussion}

The 8 stellar targets have distances ranging from $225-500$ pc and are all located within an area of $\sim 13^{\circ}$ diameter on the sky (see Fig. 6). In Fig. 7 we show their placement within the Local Chimney with respect to the neutral gas boundary of the Local Bubble cavity as recently determined by Lallement et al. (2003). From the data contained within Tables 2 and 3 we note that the vast majority of absorption components that we have detected possess negative velocities in excess of $-30 \mathrm{~km} \mathrm{~s}^{-1}$. Such values far exceed the range of velocities that may be expected from (potentially absorbing) interstellar gas that is corotating with the Galaxy at the relatively small distances presently sampled in the lower halo above the galactic plane (Fich et al. 1989). Thus, these anomalous gas velocities must arise by a mechanism other than galactic rotation. Several other authors have also noted that observations of sight-lines at high galactic latitudes generally reveal interstellar absorption at peculiar velocities (Albert et al. 1991; Danly 1989). In the following section we discuss the pattern of absorption recorded to all 8 sight-lines and forward possible explanations for the anomalous gas cloud velocities observed towards these inner halo regions. 

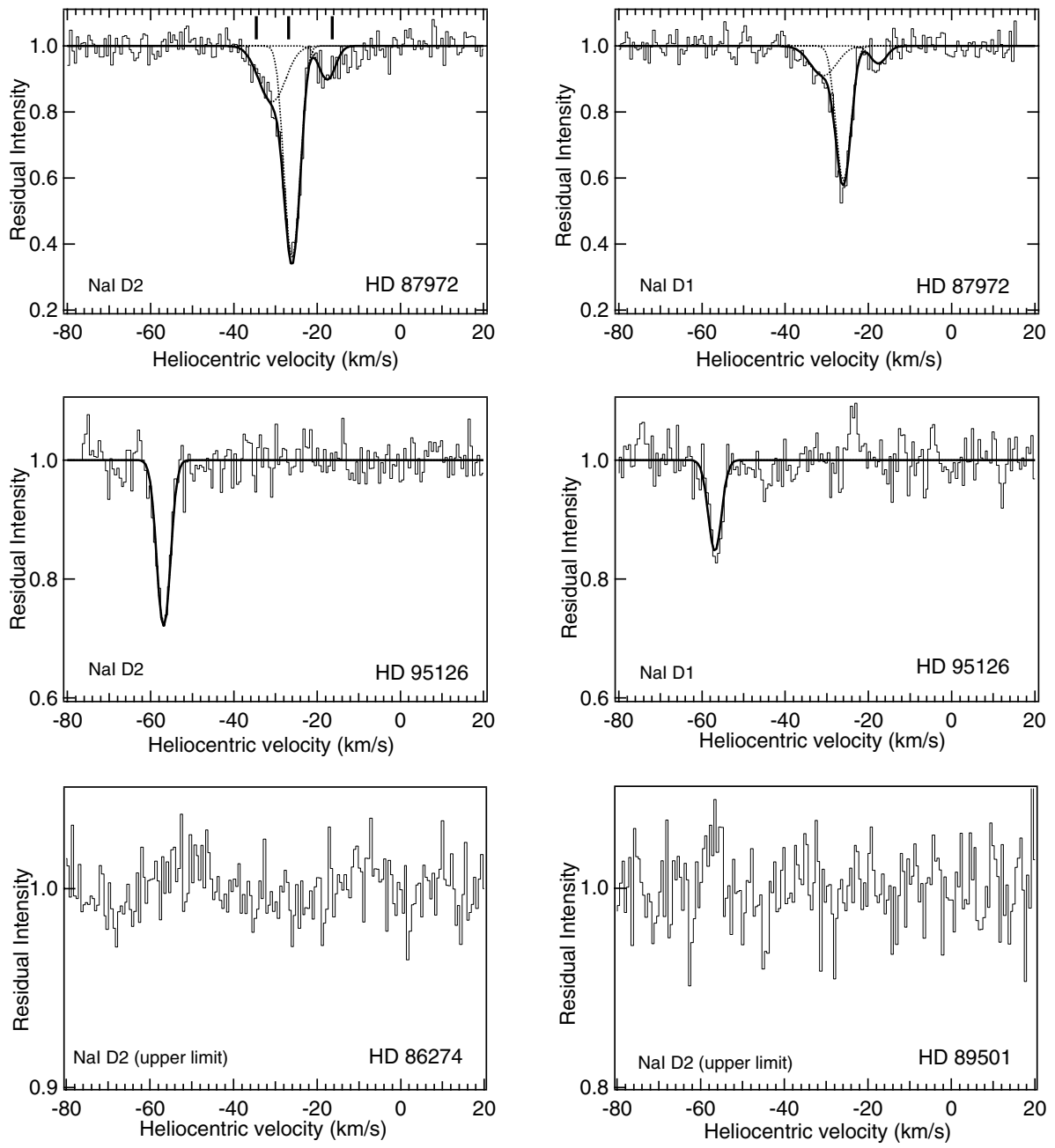

Fig. 1. Interstellar NaI D2 and D1 absorption profiles observed towards HD 87972 and HD 95126 overplotted with the best-fit models (thick line) listed in Table 2. Dotted lines indicate the placement of the individual absorption components included in the model fit. We also show the data from which upper limits for the D2 line towards HD 86274 and HD 89501 were determined.

\subsection{The Nal and Call visible absorption lines}

Our observations have only detected significant $\mathrm{NaI}$ absorption towards two targets (HD 87972 and HD 95126), both stars being at a $z$-height in excess of $275 \mathrm{pc}$. However, interstellar CaII has been detected towards 5 targets, with the most complex pattern of absorption being detected towards the most distant target, HD 88545, at a $z$-height of $380 \mathrm{pc}$. Taken as a whole these data suggest a random and patchy pattern of absorption by cold and neutral gas as a function of distance away from the galactic plane, with no detection of a dense and uniform absorption boundary that might represent an "end" to the tube-like extension of the local cavity in the inner halo. Instead, the LC seems filled with warmer and more ionized interstellar CaII gas (at least from $z \sim 185 \mathrm{pc}$ upwards).

In only two sight-lines (towards HD 87972 and HD 95126) have we been able to detect both $\mathrm{NaI}$ and CaII at a common velocity. The $N(\mathrm{NaI}) / N(\mathrm{CaII})$ ratio has been widely used as a diagnostic of the physical condition of the diffuse ISM since $\mathrm{Ca}$ is a sensitive probe of interstellar dust grain adsorption and desorption processes which, in turn, are dependent on the effects of ionization, temperature and interstellar shocks
(Barlow 1978). This ratio is presently found to be $<0.5$ for both of the common velocity gas components in the LC. Similarly low values of this ratio have been found by Crawford et al. (2002) for interstellar gas clouds lying along the opening of the southern LC into the inner galactic halo, and also for diffuse cloud(lets) located within the local cavity (Bertin et al 1993). It is argued by Crawford et al. that this low ratio is solely a result of the absence of dense (NaI-tracing) foreground clouds within the LC, that instead possess a modest depletion of Ca.

In the ultra-high resolution study of gas in the southern galactic extension of the LC, Crawford et al. (2002) generally determined narrow line-widths indicative of gas temperatures less than $2000 \mathrm{~K}$ for the observed $\mathrm{NaI}$ and CaII absorption components. Unfortunately the instrumental resolving power of the McDonald echelle is insufficient to determine the intrinsic width (and hence effective temperature) of the presently detected absorption lines. However, our best-fit $b$-values represent reliable upper limits to the temperature of the interstellar gas and its turbulence in these (unresolved) absorption components, and we see that for the majority of these components the derived best-fit $b$-values are generally $<2.5 \mathrm{~km} \mathrm{~s}^{-1}$ (i.e. $<2000 \mathrm{~K}$ ). Thus, it would appear that the gas clouds in the 

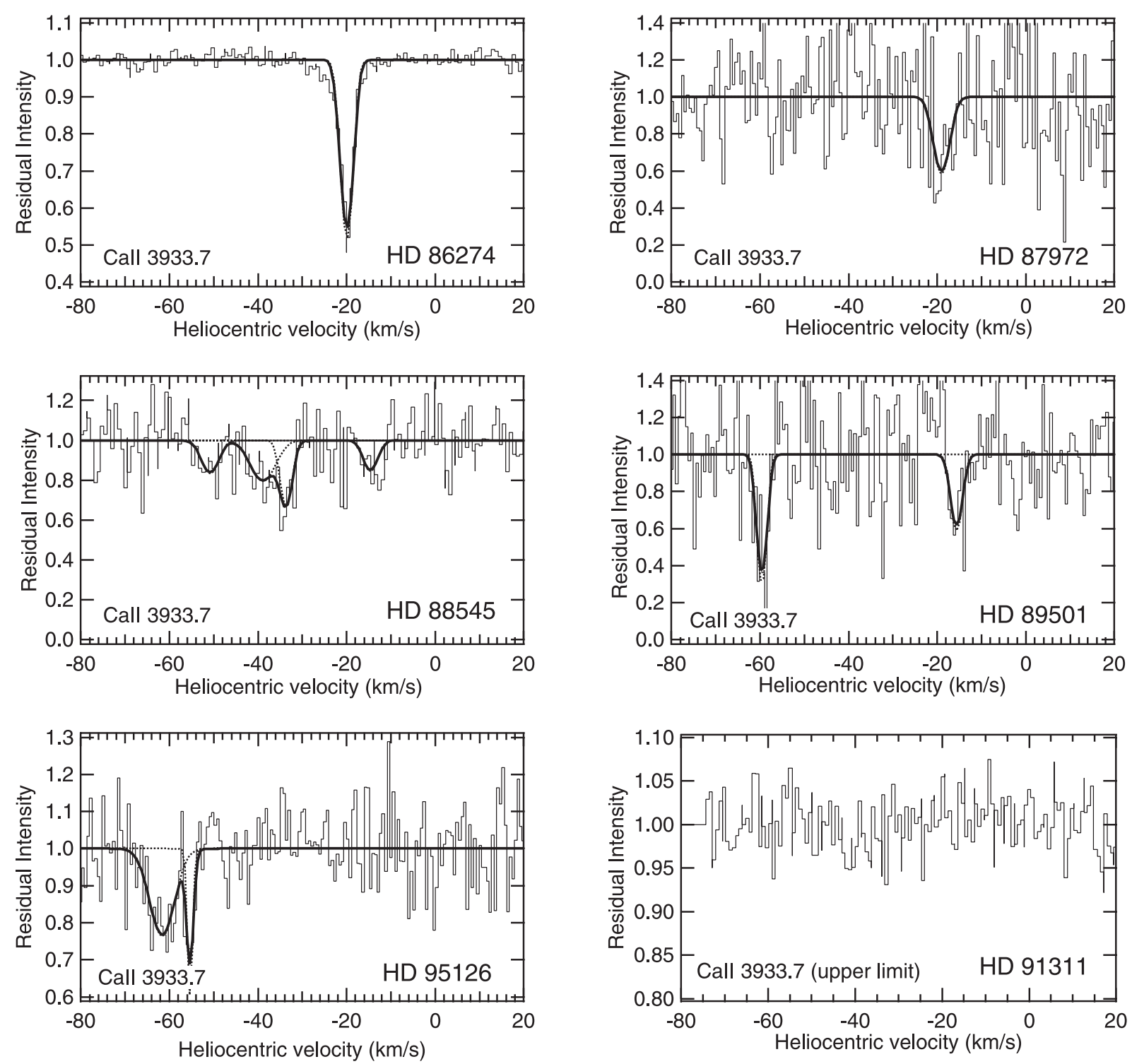

Fig. 2. Interstellar CaII K-line absorption profiles observed towards the six early-type stars lying along the Local Chimney, together with overplots of the best-fit models as listed in Table 2.

lower inner halo that are apparently falling towards the galactic disk through both openings of the LC are of a similar temperature of $<2000 \mathrm{~K}$. The narrow line-widths detected by us and by Crawford et al. are thus inconsistent with a temperature of $\sim 6000 \mathrm{~K}$ that has generally been assumed for gas residing at (the slightly higher) $z$-distances of 300-600 pc in the Lockman Layer of the halo (Lockman 1984).

The majority of the observed $\mathrm{NaI}$ and $\mathrm{CaII}$ absorption components are formed at velocities much more negative than $0 \mathrm{~km} \mathrm{~s}^{-1}$, this latter velocity being what one may have expected from absorption due to the total line-of-sight path through the local cavity. However, we do observe significant absorption at/near zero velocity in all of the UV lines listed in Table 3 (see Sect. 4.2). Taken together these results imply that the majority of the low velocity interstellar gas that purportedly fills the LC must have an ionization potential (I.P.) $>11.87 \mathrm{eV}$ (i.e. greater than that of the ionization potentials of $\mathrm{NaI}$ and $\mathrm{CaII}$ ), but less than $47.9 \mathrm{eV}$ (the I.P. of the far UV CIII line). Alternately, the column density of low velocity neutral gas in the local cavity with an I.P. $<11.87 \mathrm{eV}$ may be extremely low and could therefore be beneath our present detection limit for the $\mathrm{NaI}$ and CaII ions. In addition we note that no detections of the strong CI $\lambda 1139$ line (I.P. $=11.3 \mathrm{eV}$ ) have been reported for sightlines within 100 pc (Lehner et al. 2003; Welsh et al. 2002), which supports the view that the majority of low velocity gas in the local ISM has an I.P. $>11.87 \mathrm{eV}$ (i.e. it is mainly ionized and not neutral).

The most interesting common factor that links all of the absorption components we have presently detected in the NaI and CaII lines is that they all have negative velocities. We note that there is no single common gas cloud velocity observed in all the sight-lines, thus suggesting that we have detected several isolated infalling gas clouds. Such clouds may be physically similar to those of the type recently found by Lockman (2002) in the southern inner halo, that appear to be falling towards the galactic disk at velocities ranging from -20 to 
Table 3. Interstellar FUV absorption lines towards RE J1043+490 and RE J1059+512.

\begin{tabular}{|c|c|c|c|c|c|c|c|c|}
\hline \multirow[b]{2}{*}{ Line } & \multicolumn{3}{|c|}{ RE J1043+490 } & \multicolumn{5}{|c|}{ RE J1059+512 } \\
\hline & $\begin{array}{c}W_{\lambda} \\
(\mathrm{m} \AA)\end{array}$ & $\begin{array}{c}V \\
\left(\mathrm{~km} \mathrm{~s}^{-1}\right)\end{array}$ & $b$ & $\begin{array}{c}N \\
\left(10^{12} \text { at. } \mathrm{cm}^{-2}\right)\end{array}$ & $\begin{array}{c}W_{\lambda} \\
(\mathrm{m} \AA)\end{array}$ & $\begin{array}{c}V \\
\left(\mathrm{~km} \mathrm{~s}^{-1}\right)\end{array}$ & $b$ & $\begin{array}{c}N \\
\left(10^{12} \text { at. } \mathrm{cm}^{-2}\right)\end{array}$ \\
\hline C II $\lambda 1036.3 \ldots . .$. & $260 \pm 30$ & $\begin{array}{l}-36 \\
+4\end{array}$ & $\begin{array}{l}16 \\
18\end{array}$ & $\begin{array}{l}175 \pm 40 \\
375 \pm 50\end{array}$ & $325 \pm 35$ & $\begin{array}{l}-32 \\
+0.5 \\
+30\end{array}$ & $\begin{array}{c}18.5 \\
18.5 \\
16\end{array}$ & $\begin{array}{c}190 \pm 60 \\
>1000 * * \\
120 \pm 50\end{array}$ \\
\hline C II* $\lambda 1037.0 \ldots .$. & $<8$ & & & & $<10$ & & & \\
\hline C III $\lambda 977.0 \ldots .$. & $195 \pm 25$ & $\begin{array}{c}-39 \\
0\end{array}$ & $\begin{array}{l}18 \\
20\end{array}$ & $\begin{array}{l}21 \pm 7 \\
31 \pm 8\end{array}$ & $275 \pm 30$ & $\begin{array}{l}-27 \\
+2 \\
+20\end{array}$ & $\begin{array}{c}16 \\
9 \\
20\end{array}$ & $\begin{aligned} 42 & \pm 15^{* *} \\
7 & \pm 2 * * \\
33 & \pm 10^{* *}\end{aligned}$ \\
\hline NI $\lambda 1134.4 \ldots .$. & $40 \pm 10$ & $\begin{array}{c}-43 \\
+1\end{array}$ & $\begin{array}{l}5 \\
9\end{array}$ & $\begin{array}{c}29 \pm 5 \\
125 \pm 30\end{array}$ & $55 \pm 15$ & $\begin{array}{c}-34 \\
+2\end{array}$ & $\begin{array}{c}9 \\
11\end{array}$ & $\begin{array}{c}90 \pm 20 \\
105 \pm 40\end{array}$ \\
\hline NI $\lambda 1134.9 \ldots .$. & $55 \pm 10$ & $\begin{array}{c}-40 \\
0\end{array}$ & $\begin{array}{c}7 \\
11\end{array}$ & $\begin{array}{l}38 \pm 10 \\
95 \pm 20\end{array}$ & $90 \pm 15$ & $\begin{array}{c}-33 \\
+2\end{array}$ & $\begin{array}{l}11 \\
12\end{array}$ & $\begin{array}{c}80 \pm 20 \\
135 \pm 30\end{array}$ \\
\hline NII $\lambda 1084.0 \ldots .$. & $190 \pm 20$ & $\begin{array}{c}-37 \\
+1\end{array}$ & $\begin{array}{l}13 \\
13\end{array}$ & $\begin{array}{c}95 \pm 15 \\
260 \pm 30\end{array}$ & $265 \pm 30$ & $\begin{array}{l}-34 \\
-5 \\
+28\end{array}$ & $\begin{array}{l}17 \\
17 \\
18\end{array}$ & $\begin{array}{c}68 \pm 20 \\
220 \pm 50 \\
145 \pm 25\end{array}$ \\
\hline OI $\lambda 1039.2 \ldots .$. & $60 \pm 10$ & $\begin{array}{c}-42 \\
0\end{array}$ & $\begin{array}{c}7 \\
11\end{array}$ & $\begin{array}{c}315 \pm 50 \\
575 \pm 100\end{array}$ & $110 \pm 20$ & $\begin{array}{c}-33 \\
+1 \\
+19\end{array}$ & $\begin{array}{c}11 \\
13 \\
8\end{array}$ & $\begin{array}{c}605 \pm 130 \\
800 \pm 200 \\
260 \pm 50\end{array}$ \\
\hline $\begin{array}{l}\text { OVI } \lambda 1031.9 \\
\text { SiII } \lambda 989.9 \ldots . . .\end{array}$ & $\begin{array}{c}8 \pm 2 \\
110 \pm 20\end{array}$ & $\begin{array}{l}-3 \\
-39 \\
-2\end{array}$ & $\begin{array}{l}10 \\
13 \\
13\end{array}$ & $\begin{array}{c}8 \pm 2 \\
42 \pm 10 \\
60 \pm 20\end{array}$ & $\begin{array}{c}\text { see text } \\
145 \pm 20\end{array}$ & $\begin{array}{c}- \\
-30 \\
-1 \\
+27\end{array}$ & $\begin{array}{l}- \\
13 \\
13 \\
13\end{array}$ & $\begin{array}{c}- \\
26 \pm 5^{* *} \\
57 \pm 10^{* *} \\
31 \pm 6\end{array}$ \\
\hline PII $\lambda 1152.8 \ldots .$. & $<10$ & & & & $<12$ & & & \\
\hline ArI $\lambda 1048.2$ & $<10$ & & & & $10 \pm 3$ & -5 & 8 & $3.3 \pm 1$ \\
\hline FeII $\lambda 1063.2 \ldots .$. & $20 \pm 5$ & $\begin{array}{c}-32 \\
+2\end{array}$ & $\begin{array}{l}6 \\
8\end{array}$ & $\begin{array}{l}14 \pm 5 \\
25 \pm 6\end{array}$ & $38 \pm 10$ & $\begin{array}{c}-30 \\
0\end{array}$ & $\begin{array}{l}9 \\
8\end{array}$ & $\begin{array}{l}38 \pm 15 \\
30 \pm 10\end{array}$ \\
\hline FeII $\lambda 1144.9 \ldots .$. & $50 \pm 10$ & $\begin{array}{l}-40 \\
-2\end{array}$ & $\begin{array}{l}6 \\
6\end{array}$ & $\begin{array}{c}15 \pm 5 \\
30 \pm 10\end{array}$ & $70 \pm 15$ & $\begin{array}{l}-28 \\
+1\end{array}$ & $\begin{array}{l}9 \\
8\end{array}$ & $\begin{array}{l}38 \pm 10 \\
27 \pm 10\end{array}$ \\
\hline
\end{tabular}

$-60 \mathrm{~km} \mathrm{~s}^{-1}$. Interstellar absorption observations towards several targets within $\sim 15^{\circ}$ of $\left(l=155^{\circ}, b=+58^{\circ}\right)$ have also revealed many absorption components with a similar range of negative velocity (Lehner et al. 1999; Ryans et al. 1997). The Bell Laboratories $21 \mathrm{~cm}$ HI sky survey has revealed gas with velocities of -20 to $-60 \mathrm{~km} \mathrm{~s}^{-1}$ extending nearly $100^{\circ}$ across the sky at high latitudes in the northern galactic hemisphere (Stark et al. 1992). Kuntz \& Danly (1996) have studied the kinematics of this extensive region of intermediate velocity (IV) gas clouds, which are generally referred to as the IV Arch. They note that a key parameter that is required to evaluate the various models that address the possible origin of this large interstellar feature is the distance to the gas cloud(s). Wakker (2001) has discussed various distance estimates to each of the many sub-structures that make up the IV Arch, with a $z$-height of $0.8-1.5 \mathrm{kpc}$ generally being favored for the majority of the IV clouds. All of our presently sampled sight-lines are contained within a region on the sky (circled in white in Fig. 6) that lies between features named IV18 and IV21, within an area that has a low HI column density, $N(\mathrm{HI})<5 \times 10^{19} \mathrm{~cm}^{-2}$, for gas with velocities lying between -30 and $-60 \mathrm{~km} \mathrm{~s}^{-1}$. Ryans et al. (1997) have observed high-resolution $\mathrm{NaI}$ and CaII interstellar lines towards the star AG $+53783\left(l=154.4^{\circ}, b=+56.6^{\circ}\right)$, which also lies within this circle and recorded absorption components at
-63 and $-47 \mathrm{~km} \mathrm{~s}^{-1}$. These velocities are within $\pm 5 \mathrm{~km} \mathrm{~s}^{-1}$ of the absorption components presently observed by us towards HD 88545, HD 89501 and HD 95126. Due to an uncertain spectral classification for AG +53 783 Ryans et al. could only estimate its $z$-distance to be $0.4 \mathrm{kpc}$. This seems consistent with the $z$-distances of $0.380 \mathrm{kpc}$ for HD $88545,0.320 \mathrm{kpc}$ for HD 89501 and $0.380 \mathrm{kpc}$ for HD 95126. Therefore, based on these 4 detections of similar intermediate-velocity gas components (with $V \sim-55 \mathrm{~km} \mathrm{~s}^{-1}$ ) and the non-detection of such components towards HD $87972(z=275 \mathrm{pc})$ we can now provide a probable $z$-distance of $275-320$ pc to this part of the IV Arch. This places the distance to these IV clouds much closer than previously thought and in Sect. 4.4 we discuss their possible link to the origin of the Local Chimney.

\subsection{The far UV absorption spectra of RE J1043+490 and $R E J 1059+512$}

We have observed the far UV interstellar spectra towards two hot white dwarf stars, RE J1043+490 ( $T \sim 47500 \mathrm{~K})$ and RE J1059+512 $(T \sim 68500 \mathrm{~K})$ with respective distances of $230 \mathrm{pc}$ and $315 \mathrm{pc}$ (Vennes et al. 1997). Thus, both of their sight-lines sample the gas in the LC that is foreground to several of the more distant targets sampled by our NaI and CaII observations. We have detected only 10 far UV interstellar lines 

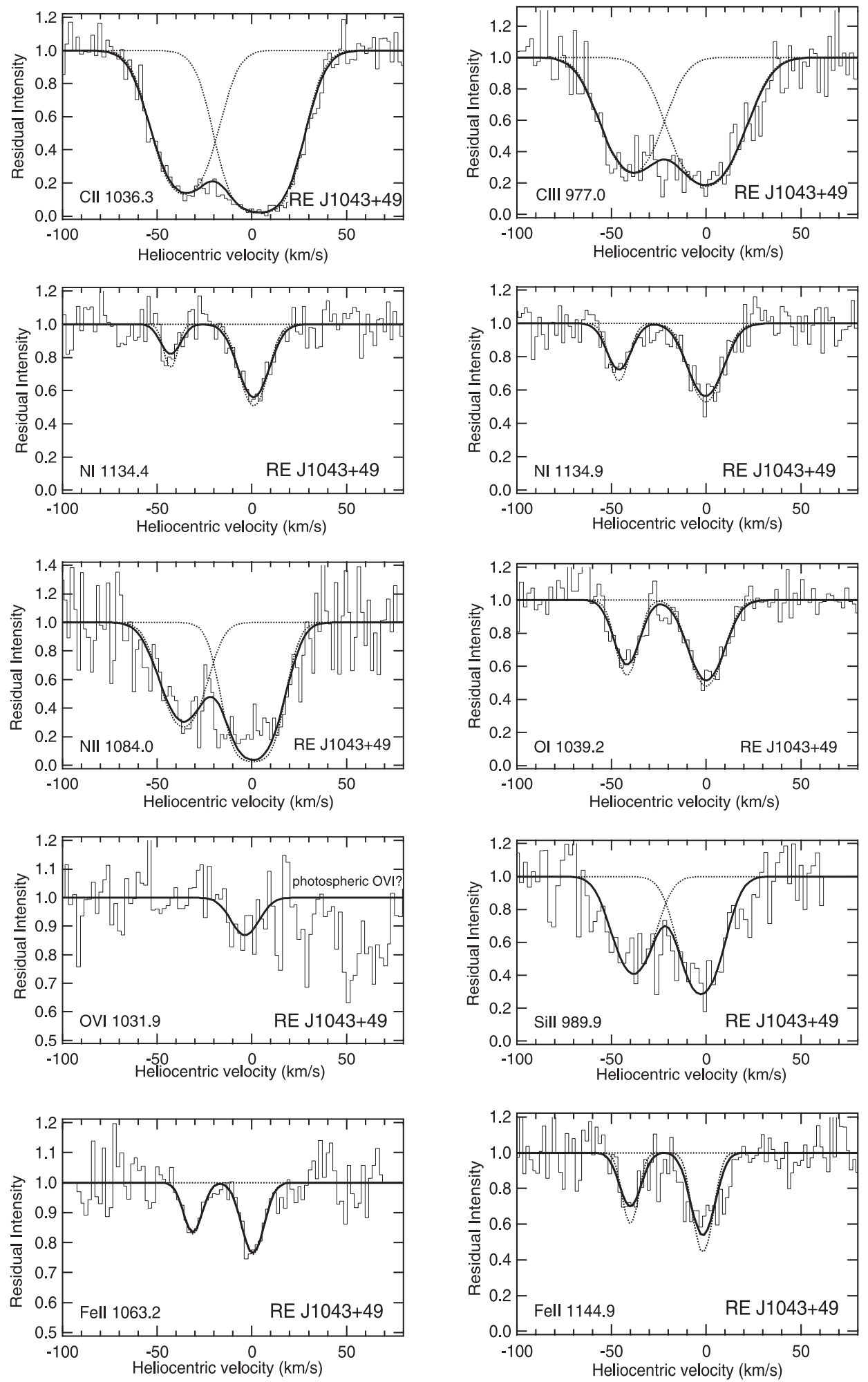

Fig. 3. Far UV interstellar absorption line-profiles observed towards RE J1043+490 together with their best-fit models as listed in Table 3.

towards RE J1043+490 and 11 lines towards the more distant white dwarf, suggesting that we are sampling a very low density phase of the interstellar medium. This is consistent with the findings of Marsh et al. (1997) who have estimated a total neutral hydrogen column density of only $N(\mathrm{HI})=1.7 \times 10^{19} \mathrm{~cm}^{-2}$ towards RE J1043+490. Also, based on the detectability of RE J1059+512 by the Extreme Ultraviolet Explorer satellite, a similarly low value of $N(\mathrm{HI})<5 \times 10^{19} \mathrm{~cm}^{-2}$ seems appropriate for that sight-line.

Based on the ionization potentials of the far UV spectral lines that have been detected towards both targets, appreciable levels of ionized interstellar (HII) gas must be present. Holberg et al. (1999) have estimated that $N(\mathrm{HI}) \sim N(\mathrm{HII})$ for the $110 \mathrm{pc}$ sight-line towards the star RE J1032+532, 

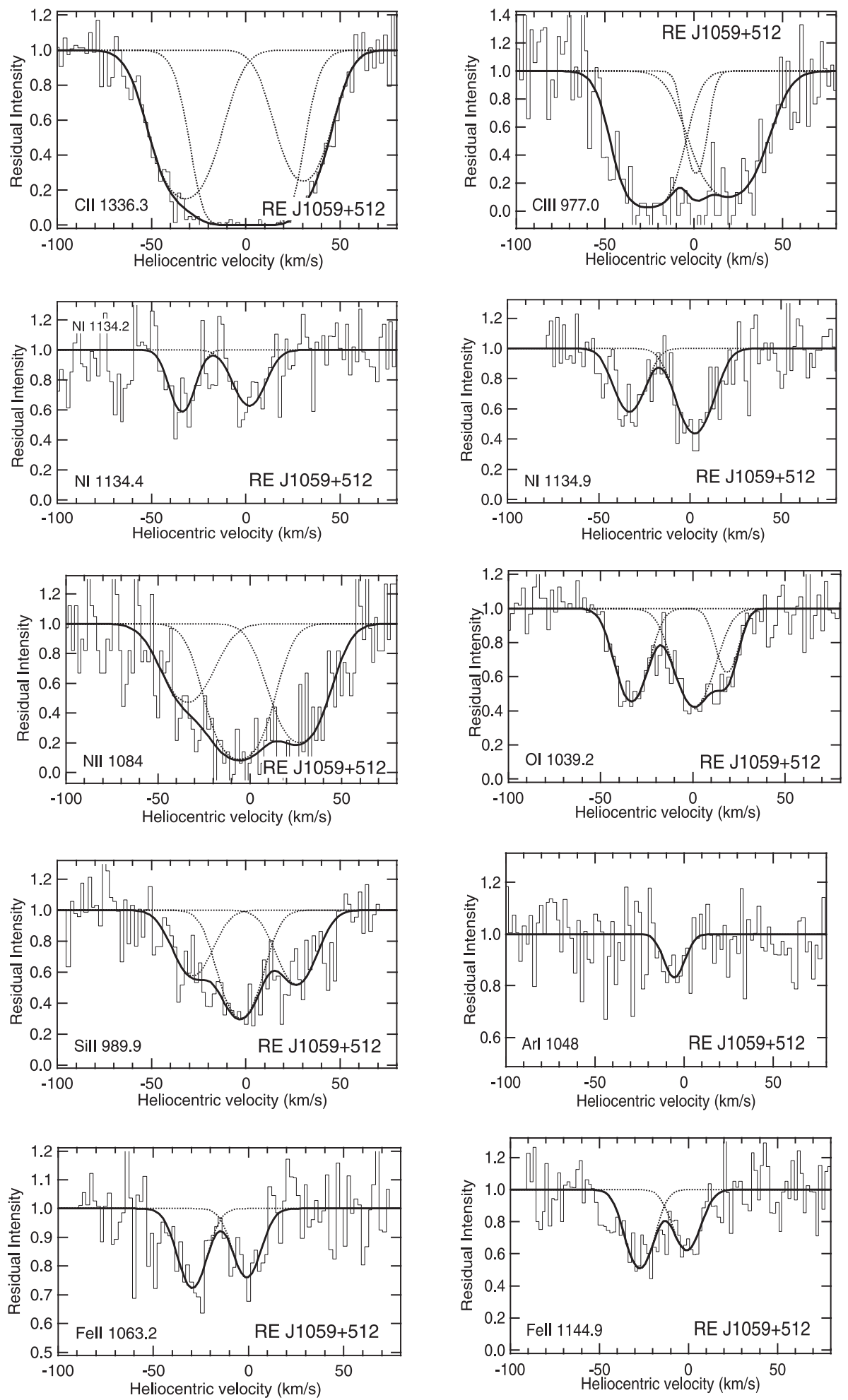

Fig. 4. Far UV interstellar absorption line-profiles observed towards RE J1059+512 together with their best-fit models as listed in Table 3.

which also lies within the $\mathrm{LC}$ and is only $\sim 5^{\circ}$ away from both of the white dwarfs presently observed. In their study of interstellar gas towards 30 sight-lines within 200 pc, Lehner et al. (2003) found that both the CII $\lambda 1036$ and the CIII $\lambda 977$ lines (which are good traces of an ionized medium) were ubiquitous, with a $N(\mathrm{CIII}) / N(\mathrm{CII})$ ratio typically of $\sim<-1.0$ dex. We derive similar values of $-1.02 \mathrm{dex}$ and $<-1.4 \mathrm{dex}$ for the
RE J1043+490 and RE J1059+512 sightlines respectively. Thus, CII is the dominant ion in the local ISM, again supporting the view outlined in Sect. 4.1 that we are sampling a mainly warm and ionized medium. In a recent HI, UV and Xray study of the emission from interstellar gas associated with the Lockman Hole, Kappes et al. (2003) found that their data was best fit under the assumption that a large fraction of the 


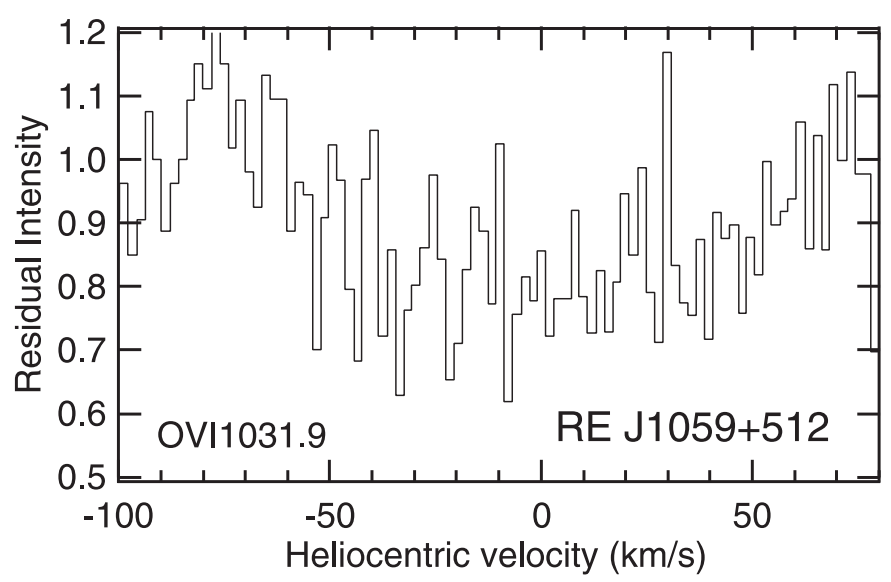

Fig. 5. OVI $\lambda 1032 \AA$ profile recorded towards RE J1059+512. The width of the absorption profile suggests a stellar, rather than an interstellar, origin

attenuation of the soft X-ray emission is caused by ionized interstellar hydrogen gas. They concluded that the majority of neutral and molecular gas observed in this galactic direction was most probably located in the lower halo, rather than in the disk component. This view is supported by our NaI observations which only detect cold and neutral gas at $z$-distances $>275 \mathrm{pc}$.

We have been unable to detect any of the strong UV absorption lines of molecular $\mathrm{H}_{2}$ in either of the white dwarf spectra. This is not surprising, since the survey by Lehner et al. (2003) only managed to detect $\mathrm{H}_{2}$ gas at the (dense) boundary of the local cavity. This is consistent with the sight-lines towards both white dwarfs being contained within any neutral boundary that may exist to the LC.

The results listed in Table 3 show that the majority of the UV absorption lines detected towards both white dwarfs can be fit with a two-component model. In the case of RE J1043+490 the main (zero velocity) absorption component is accompanied by an additional component at $V \sim-39 \mathrm{~km} \mathrm{~s}^{-1}$, and similarly for $\mathrm{RE} \mathrm{J} 1059+512$ the main component requires additional absorption at $V \sim-31 \mathrm{~km} \mathrm{~s}^{-1}$. This two-component structure can be easily recognized in the interstellar profiles of the FeII $\lambda 1144.9$ and NI $\lambda 1134.9$ lines shown in Figs. 3 and 4. Unfortunately we do not have information on the corresponding values of $N(\mathrm{HI})$ for each of these clouds, but under the following assumptions we can derive reasonable estimates for the elemental abundances of the gas observed towards each hot white dwarf. Based on the findings of Holberg et al. (1999) and Marsh et al. (1997), we assume that there are at least equal amounts of $N(\mathrm{HII})$ and $N(\mathrm{HI})$ in the LC. Therefore, we assume a total of $\log N(\mathrm{HI}+\mathrm{HII})=19.54 \mathrm{~cm}^{-2}$ towards RE J1043+490. We note that our derived column density values for OI and NI (which generally trace the neutral HI gas) are $\sim 50 \%$ higher towards RE J1059+512, and therefore we assume a total of $\log N(\mathrm{HI}+\mathrm{HII})=19.72 \mathrm{~cm}^{-2}$ for this sightline. These values of total hydrogen column density are both consistent with the low column density values found towards the Lockman Hole region and are also consistent with the levels of absorption predicted by the observed level of soft X-ray emission.

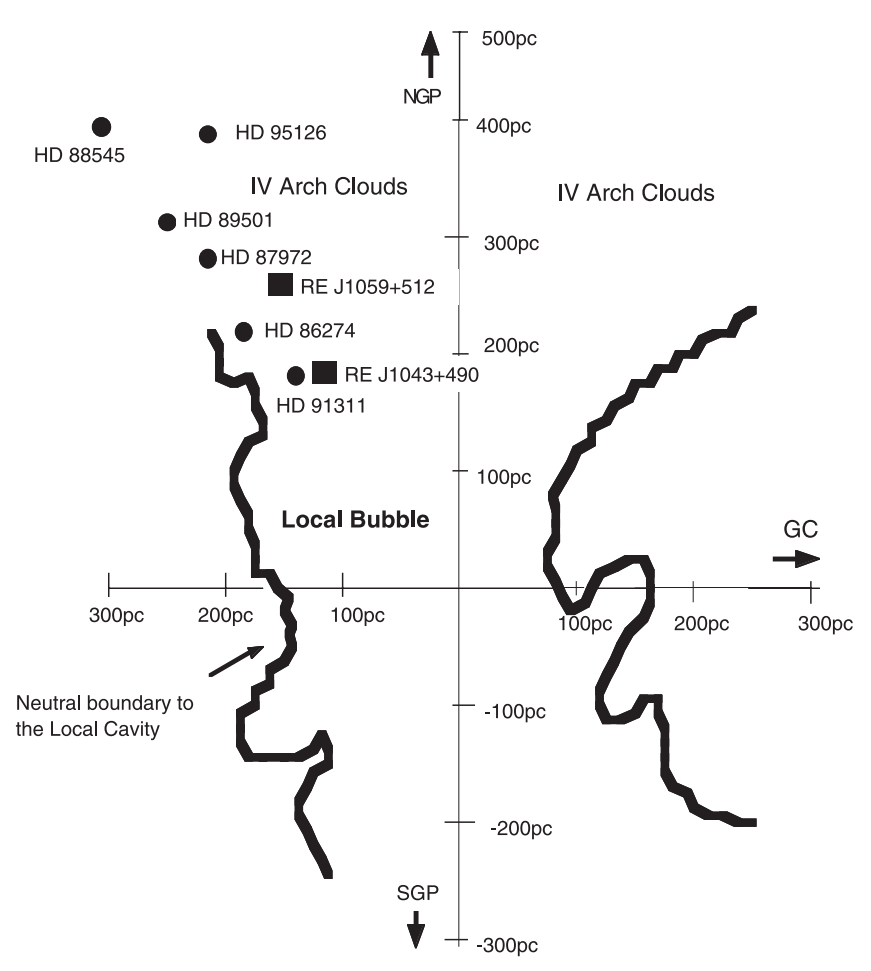

Fig. 6. Positions of the 8 target stars with respect to the cold and dense neutral gas boundary to the Local Bubble cavity (solid line) as mapped by Lallement et al. (2003) using NaI absorption measurements towards 1005 sight-lines in the local ISM. Large dots represent targets presently observed at $\mathrm{NaI}$ and CaII, large squares represent the location of the two hot white dwarf stars observed with FUSE.

Using the derived values of column density listed in Table 3, and assuming that these are the dominant state of these elements in the local interstellar gas (which may not be the case for $\mathrm{O}$ ), we calculate their abundance ratios relative to that of $\mathrm{H}$. These values are listed in Table 4. We note that although both $\mathrm{OI}$ and NI ions are tightly coupled to HI (neutral) gas by charge exchange reactions, they can also exist in ionized (HII) regions (Sembach \& Savage 1996). For nitrogen we have detected lines from both NI and NII states and thus our derived abundance of $\mathrm{N}$ relative to $N(\mathrm{H})$ is probably accurate. However, for oxygen and argon only the neutral lines are available and thus our derived abundances for $\mathrm{O}$ and $\mathrm{Ar}$ (which ignores any contribution from OII and ArII) are only an approximation. We estimate that ignoring the effects of ionization from these ions results in an error of $\sim \pm 0.2$ dex in their derived abundances.

Our main finding is that all the derived elemental abundance values are very similar for both sight-lines, thus indicating that the gas clouds encountered in the LC have very similar physical and chemical properties. Most of these abundance values (with the exception of Ar and O) are within \pm 0.3 dex of those found by Holberg et al. (1999) and Welsh et al. (2002) for the interstellar gas within the LC, which is found to be moderately depleted. The variation in the relative elemental abundance ratio of $\mathrm{OI} / \mathrm{HI}, \mathrm{NI} / \mathrm{HI}$ and $\mathrm{ArI} / \mathrm{HI}$ in the local cavity has recently been investigated using the FUSE satellite by Moos et al. (2002) and Jenkins et al. (2000). We derive values for these three ratios that are $\sim 0.9$ dex lower than the values 
Table 4. Element abundance estimates.

\begin{tabular}{ccccccc}
\hline \hline Sight-Line & $\log (\mathrm{C} / \mathrm{H})$ & $\log (\mathrm{N} / \mathrm{H})$ & $\log (\mathrm{O} / \mathrm{H})$ & $\log (\mathrm{Si} / \mathrm{H})$ & $\log (\mathrm{Ar} / \mathrm{H})$ & $\log (\mathrm{Fe} / \mathrm{H})$ \\
\hline RE J1043+490 & -4.7 & -4.9 & -4.6 & -5.5 & N/A & -5.9 \\
RE J1059+512 & $<-4.6$ & -4.9 & -4.5 & -5.7 & -6.7 & -5.9 \\
\hline
\end{tabular}

found by those authors. We attribute this abundance difference to a variation in the local intensity of ionizing radiation present along the LC that affects the ionization balance of OI, ArI and NI (i.e. a significant fraction of these elements reside in higher ionization states, as confirmed by our detection of interstellar NII towards both hot white dwarfs).

Finally we note that since there is some uncertainty in the absolute velocity of the absorption components detected in the far UV lines, there remains a possibility that the main component formed towards both white dwarfs should not be placed at zero velocity. We note that if, instead, we had assumed a velocity of $V_{\text {helio }}=-15 \mathrm{~km} \mathrm{~s}^{-1}$ for the main components towards both stars then the higher velocity components would have been formed at $-54 \mathrm{~km} \mathrm{~s}^{-1}$ for RE J1043+490 and -46 $\mathrm{km} \mathrm{s}^{-1}$ for RE J1059+512. Such velocities are similar to those found in the $\mathrm{NaI}$ and CaII data for sight-lines with a $z$-height $>275 \mathrm{pc}$ that pass through the IV Arch clouds. Therefore, also taking into account the associated uncertainty in the white dwarf distance estimates, these two sight-lines could be passing through the very lower extremities of the IV Arch clouds.

\subsection{The highly ionized gas in the Local Chimney}

In a survey of 25 sight-lines towards hot white dwarf stars located within $200 \mathrm{pc}$ of the Sun using the FUSE satellite, Oegerle et al. (2003) found that the level of interstellar OVI $(\lambda 1031.93 \AA)$ is generally weak, and in many cases completely absent along these nearby sight-lines. The authors found an average OVI space density of $2.4 \times 10^{-8} \mathrm{~cm}^{-3}$ for the local interstellar gas, which is similar to that value found in the Galactic disk measured over kpc scales. Welsh et al. (2002) confirmed this local paucity of hot and highly ionized OVI gas by placing an upper limit of $\log N(\mathrm{OVI})<13.0 \mathrm{~cm}^{-2}$ to the column density measured towards the hot white dwarf RE J1032+532, which lies at a distance of $116 \mathrm{pc}$ along the Local Chimney. Our present observations have detected absorption features at wavelengths close to that of the important OVI $\lambda 1032 \AA$ line in both of the white dwarf spectra. Such features are also detected, with about half the strength, in the weaker member of the OVI doublet at 1037.6 $\AA$. It has recently been discovered that some hot white dwarf stars with temperatures as low as $45000 \mathrm{~K}$ can produce photospheric OVI lines (Chayer et al. 2003), and thus there must remain some uncertaintly as to whether these absorption features are of a stellar or interstellar origin. Unfortunately the stellar radial velocities for both hot white dwarf stars are unknown, and neither of their far UV spectra contain detectable features that might be associated with the expectedly strong photospheric lines of PII at $\lambda 1030.5 \AA$ or CIII at $\lambda 1174.9 \AA$.

Inspection of the OVI absorption profile for RE J1043+490 (shown in Fig. 3) reveals two absorption features; one narrow line centered close to $V=0 \mathrm{~km} \mathrm{~s}^{-1}$ and a possible broad feature centered $\sim+50 \mathrm{~km} \mathrm{~s}^{-1}$. Since we have also detected interstellar absorption near zero velocity in many other far UV lines towards this star (see Table 3), it seems likely that the zero velocity OVI feature is also of interstellar origin. Given the anomalous positive velocity of the (noisy) broad OVI absorption feature, we tentatively identify this with being of photospheric origin. Additionally, we derive an interstellar column density, $N(\mathrm{OVI})=7 \times 10^{12} \mathrm{~cm}^{-2}$, along the $230 \mathrm{pc}$ path length to RE J1043+490 which is only $\sim 30 \%$ greater than that predicted by the average OVI space density derived from the FUSE OVI survey of the local ISM.

The OVI absorption profile measured towards RE J1059+512 (shown in Fig. 5) is even more problematic to understand. We detect a very broad and noisy absorption profile extending over a velocity range of $\sim 100 \mathrm{~km} \mathrm{~s}^{-1}$, which would strongly suggest a stellar origin. The equivalent width of this line is $\sim 50 \mathrm{~m} \AA$ and a model fit to the profile requires an unrealistically large doppler $b$-value with a correspondingly very high value of OVI interstellar column density. Alternatively, the measured profile could conceivably consist of an unresolved blend of both interstellar and stellar components, and thus without knowledge of the stellar radial velocity of the hot white dwarf star we are at present unable to assess the level of any interstellar contribution to this absorption profile.

The OVI $\lambda 1032 \AA$ line has been detected in emission towards a sight-line only $\sim 20$ arcsec from the direction towards RE J1043+490 (Welsh et al. 2002). Emission was detected at a velocity of $V_{\text {helio }}=-20 \pm 22 \mathrm{~km} \mathrm{~s}^{-1}$ at a level of $2500 \pm$ 700 photons $\mathrm{cm}^{-2} \mathrm{~s}^{-1} \mathrm{sr}^{-1}$ (LU). Although the presently detected OVI absorption occurs at a velocity of $V_{\text {helio }}=$ $-3 \mathrm{~km} \mathrm{~s}^{-1}$, which lies just within the velocity range of the OVI emission, we deem it unlikely that the same spatially coincident gas cloud is responsible for both the observed OVI emission and absorption. We believe it more likely that the OVI emission is associated with more distant infalling IV gas, based on the ubiquitous amounts of intermediate negative velocity gas (as observed by ourselves and the Bell Labs HI survey) in this galactic direction. Support for this conjecture comes from the recent survey by Savage et al. (2003) in which the observed velocity of OVI absorption was found to mimic that of many IVCs as traced by their HI velocity. Clearly future observations of the high ions of CIV and SiIV in sight-lines toward and through the IV Arch would help clarify the ionization state of these inner halo clouds.

\subsection{The Local Chimney and a possible link with the Intermediate Velocity Clouds}

Our present visible and far UV absorption observations, together with many previous $\mathrm{HI}$ and UV observations 


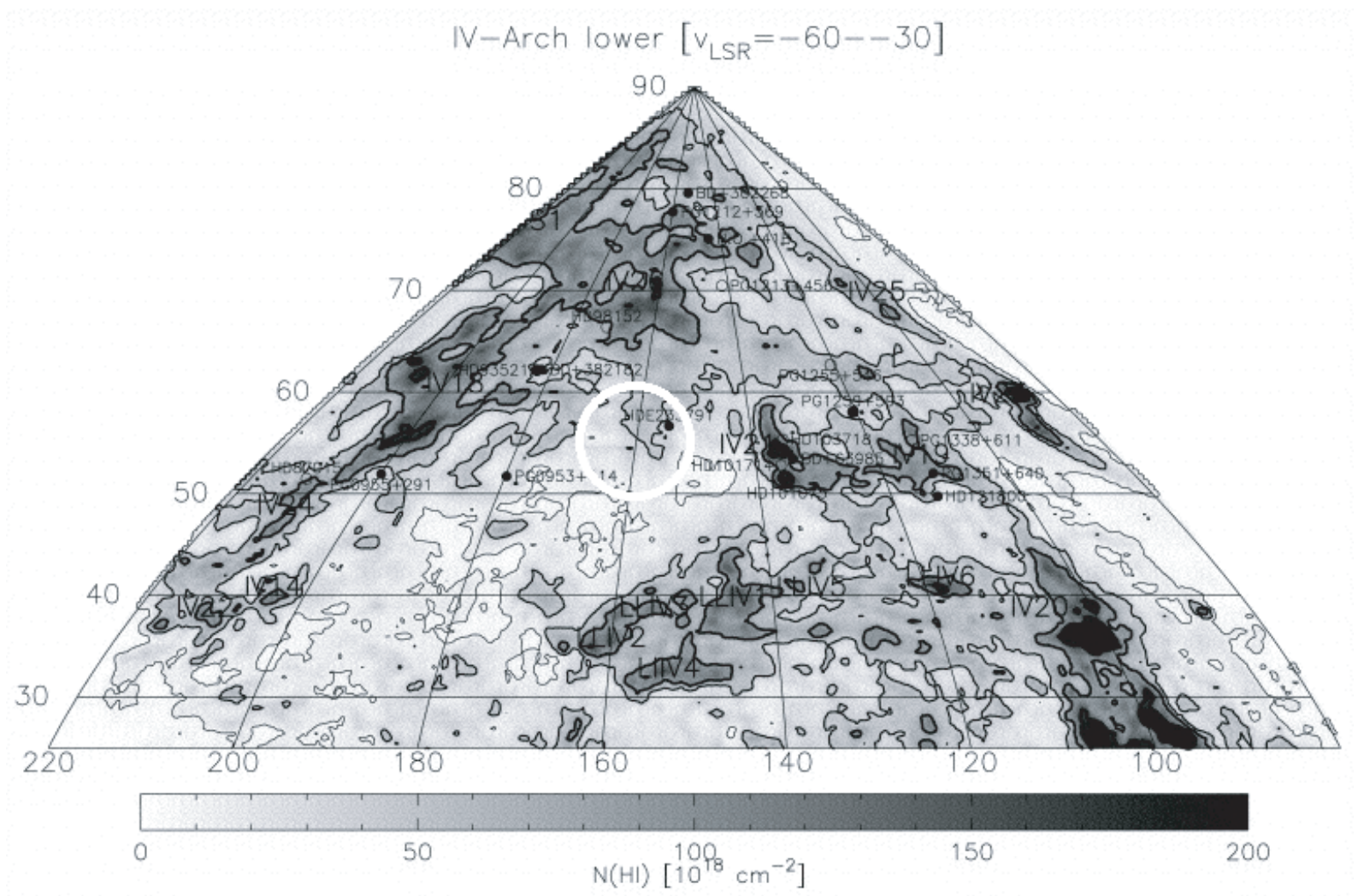

Fig. 7. Map of the IV Arch complex of interstellar gas clouds with velocities between -60 and $-30 \mathrm{~km} \mathrm{~s}^{-1}$, from Wakker (2001). The white circle represents the area on the sky presently sampled by our absorption observations.

(Wakker 2001), have confirmed the presence of interstellar gas with velocities ranging from -20 to $-60 \mathrm{~km} \mathrm{~s}^{-1}$ that extends across much of the northern galactic hemisphere. Although our new observations have helped place a lower limit to the $z$-distance to the IV Arch of 275-320 pc, the question of the origin and evolution of IVCs still remains open. Kuntz \& Danly (1996) list six classes of model that have been forwarded to explain the origin of IVCs: high- $z$ gas infall, collisions of halo gas with the galactic disk, gas liberated by high- $z$ supernovae, displaced superbubble gas, gas from galactic fountains and gas associated with a high- $z$ extension of spiral arms. The "galactic fountain" model of Shapiro \& Field (1976), in which hot gas is ejected from the disk into the overlying halo by a supernova explosion can account for many of the observed kinematic and chemical properties for several IVCs (Richter et al. 2001; Kuntz \& Danly 1996). We note that there is a significant enhancement in the number of IVCs in the northern galactic polar region with respect to the southern galactic hemisphere (Wakker 2001), and that the direction of the "opening" of the Local Chimney into the inner halo points towards this region of IVC enhancement. It is therefore tempting to link the possible ejection of gas by some past explosive event that occured in the underlying galactic disk with the numerous IV Arch clouds that have since cooled and are now falling back towards the galactic plane. Verschuur (1993) has proposed that the expansion of a supershell in the Perseus Arm, that is now impacting and squeezing the local cavity, may be linked to the formation of the overlying IVCs. More recently Perrot \& Grenier (2003) have suggested that the Gould Belt could be the remnant of a large gamma-ray burst event that occured in the disk of our local region of the galaxy. Lallement et al. (2003) have noted that there seems to be many IVCs above the LC and have raised the possibility of a link between the Gould Belt, the falling IVCs and the underlying Local Bubble cavity. Interestingly, Knude \& Hog (1999) have noted unusually low amounts of neutral gas with normal levels of associated dust for sight-lines $<200$ pc towards the north galactic pole and IV Arch, which is what may be expected if gas that was once associated with the LB cavity was expelled by a sudden increase in pressure caused by a large gamma-ray burst which left interstellar dust behind. Knude \& Hog (1999) also suggest that IV gas should be present at a distance of 200-300 pc in the IV Arch direction.

Clues to the origin of IVCs can also come from their present-day gas chemistry. The metallicity of IV Arch gas has been determined in three directions towards HD 93521 (Spitzer \& Fitzpatrick 1993), HD 121800 (Van Steenberg \& Shull 1988) and towards PG 1259+593 (Richter et al. 2001). These observations suggest a near-solar abundance for the IV Arch gas, in common with the refractory element enriched diffuse clouds found throughout the galactic halo (Sembach \& Savage 1996). This abundance enhancement strongly suggests that interstellar dust grain destruction has taken place, perhaps by a previous source of strong heating such as a supernova event. In contrast, we note that the interstellar gas associated with the cloudlets located within the local cavity are substantially metal-depleted (Kimura et al. 2003).

Finally we note that although galactic fountain models (such as the low-temperature model of Houck \& Bregman 1990) can account for the kinematic behavior of infalling lower halo gas clouds, such models also predict an outflow 
(or upwelling) of hot gas from the galactic disk. The ions of CIII, CII, NII and SiII that we have observed towards both hot white dwarfs in the LC do show gas absorption at positive velocities (up to $V=+50 \mathrm{~km} \mathrm{~s}^{-1}$ ), whereas the lower ionization lines tend to exhibit mainly negative velocity features. Our sampling of the more distant (IV) gas, through the low ionization lines of $\mathrm{NaI}$ and CaII, only reveal absorption at negative velocity. As stated in Sect. 4.3, future UV observations of the higher ionization gas that may be present towards the IV Arch clouds at a $z$-height of 275-600 pc could provide evidence for the predicted outflow of the hot fountain gas into the halo. In addition, obtaining elemental abundances of these inner-halo IV gas clouds as a function of $z$-height could provide important insights into their origin and subsequent evolution.

\section{Conclusion}

We have obtained high- resolution $\left(1.7 \mathrm{~km} \mathrm{~s}^{-1}\right)$ spectra of the interstellar NaI CaII absorption lines seen towards 6 stars lying along the Local Chimney (LC) with distances ranging from 225 to $500 \mathrm{pc}$. We have also obtained far- ultraviolet absorption spectra of two hot white dwarfs, also lying along the LC with distances of $230 \mathrm{pc}$ and $315 \mathrm{pc}$, using the NASA FUSE satellite. These observations have revealed interstellar gas clouds with velocities in the $V_{\text {helio }}=-20$ to $-60 \mathrm{~km} \mathrm{~s}^{-1}$ range that appear to be falling from the inner halo onto the galactic disk. In particular, we find that gas with a velocity of $V_{\text {helio }} \sim-55 \mathrm{~km} \mathrm{~s}^{-1}$ can be associated with an intermediate velocity cloud complex called the IV Arch, whose probable $z$ height is now constrained to be $275-320 \mathrm{pc}$, which is much lower than previously thought.

Our NaI observations indicate that there is very little cold $(T<100 \mathrm{~K})$ and neutral gas contained within the first $250 \mathrm{pc}$ of the LC, whereas interstellar CaII clouds are detected with a far greater frequency. In the few cases where both $\mathrm{CaII}$ and $\mathrm{NaI}$ are detected with similar velocities the NaI/CaII column density ratio is $<0.5$, which is similar to that found for cloudlets within the local ISM. We have failed to detect any neutral boundary that defines a plausible "end" to the LC as it enters into the inner galactic halo, and our data strongly support the notion that the LC is filled with significant amounts of warm and ionized gas. Our far UV absorption observations, that sample the ISM up to a $z$-height of $\sim 270 \mathrm{pc}$, also reveal the presence of ionized gas in the LC, typically with an ionization potential of between $11.9 \mathrm{eV}$ and $48 \mathrm{eV}$. The sight-lines to both hot white dwarfs reveal two absorbing clouds, one near zero $\mathrm{km} \mathrm{s}^{-1}$ and the other separated by $-39 \mathrm{~km} \mathrm{~s}^{-1}$ (towards RE J1043+490) and $-31 \mathrm{~km} \mathrm{~s}^{-1}$ (towards RE J1059+512). Due to uncertainties in both the absolute velocity of these clouds and the distances to the how white dwarfs, there is a possibility that these two sight-lines pass through the very lower regions of the IV Arch complex.

We have derived averaged sight-line abundances towards both white dwarfs for the elements of C, N, O, Si, Ar and Fe. The abundance patterns are very similar for both sight-lines, indicating that the clouds within the LC (and the local cavity) have similar physical and chemical properties i.e. significant metal depletion. We have also detected weak interstellar
OVI $(\lambda 1032 \AA)$ absorption towards RE J1043+490, with a column density of $N(\mathrm{OVI})=7 \times 10^{12} \mathrm{~cm}^{-2}$ and a velocity of $\sim$ zero $\mathrm{km} \mathrm{s}^{-1}$. This value is consistent with recent detections of this high ion in the local ISM by FUSE.

Finally, we discuss possible origins for the IVC's observed in the northern galactic hemisphere. We note the apparent coincidence of the direction of the opening of the LC into the lower halo and the presence of low $z$ interstellar gas clouds associated with the IV Arch that are falling towards the disk. These new data may provide a causal link between the Gould Belt, the IVCs and the Local Bubble/Local Chimney structures. It is suggested that such a causal link could be confirmed by future UV absorption measurements of more gas clouds with a $z$-height of $275-600 \mathrm{pc}$ in this galactic direction.

Acknowledgements. We are grateful to the staff and directorate of the McDonald Observatory in Texas who gave invaluable assistance during our observations. In particular we are indebted to both Prof. David Lambert (University of Texas, Austin) and Prof. Steve Federman (University of Toledo) who trained us in use of the instrumentation. We would also wish to thank the FUSE mission operations staff at the Johns Hopkins University. Funding for this work was provided in part by the NASA FUSE Guest Investigator Program under the NASA contract NAS5-32985.

\section{References}

Albert, C. E., Blades, C., Morton, D. C., et al., 1993, ApJS, 99, 81

Barlow, M. 1978, MNRAS, 183, 417

Bertin, P, Lallement, R., Ferlet, R., \& Vidal-Madjar, A. 1993, A\&A, 278, 549

Chayer, P., Oliveira, C., Dupuis, J., Moos, H. W., \& Welsh, B. Y. 2003 , A\&A, in press

Crawford, I., Lallement, R., Price, R., et al. 2002, MNRAS, 337, 720

Danly, L. 1989, ApJ, 342, 785

Dickey, J. M., Weisberg, J., Rankin, J., \& Boriakoff, V. 1981, A\&A, 101,332

Dyson, J., \& Williams, D. 1997, The Physics of the Interstellar Medium, 2nd ed., ed. J.E. Dyson \& D.A. Williams (Bristol: Institute of Physics Publishing), ISBN: 0750303069

ESA 1997, The Hipparcos Satellite Catalogue, ESA SP-1200

Fich, M., Blitz, L., \& Stark, A. 1989, ApJ, 342, 272

Frisch, P. 1995, Space Sci. Rev., 72, 499

Hausen, N., Reynolds, R., Haffner, L., \& Tufte, S. 2002, ApJ, 565, 1060

Holberg, J., Bruhweiler, F., Barstow, M., \& Dobbie, P. 1999, ApJ, 517, 841

Houck, J., \& Bregman, J. 1990, ApJ, 352, 506

Howk, J. C., \& Savage, B. 1999, ApJ, 517, 746

Jenkins, E., Oegerle, W., Gry, C., Vallerga, J. V., et al. 2000, ApJ, 538, L81

Jenkins, E. B. 2002, ApJ, 580, 938

Kappes, M., Kerp, J., \& Richter, P. 2003, A\&A, 405, 607

Kimura, H., Mann, I., \& Jessberger, E. 2003, ApJ, 582, 846

Knude, J., \& Hog, E. 1999, A\&A, 341, 451

Kuntz, K., \& Danly, L. 1996, ApJ, 457, 703

Lallement, R., Bertin, P., Chassefiere, E., \& Scott, N. 1993, A\&A, 271, 734

Lallement, R., Welsh, B. Y., Vergely, J. L., Crifo, F., \& Sfeir, D. 2003, $\mathrm{A} \& \mathrm{~A}$, in press 
Lehner, N., Sembach, K., Lambert, D., Ryans, R., \& Keenan, F. 1999, Savage, B., Sembach, K., Wakker, B., Richter, P., et al. 2003, ApJS, A\&A, 352, 257

Lehner, N., Jenkins, E., Gry, C., et al. 2003, ApJ, submitted

Lockman, F. 1984, ApJ, 283, 90

Lockman, F. 2002, ApJ, 580, L47

Lockman, F., Jahoda, K., \& McCammon, D. 1986, ApJ, 302, 432

Maiz-Apellaniz, J. 2001, ApJ, 560, L83

Marsh, M., Barstow, M., Buckley, D., et al. 1997, MNRAS, 287, 705

McKee, C., \& Ostriker, J. 1977, ApJ, 218, 148

Moos, H. W., Cash, W., Cowie, L., et al. 2000, ApJ, 538, L1 146, 125

Sembach, K., \& Savage, B. 1996, ApJ, 457, 211

Sfeir, D., Lallement, R., Crifo, F., \& Welsh, B. Y. 1999, A\&A, 346, 785

Shapiro, P., \& Field, G. 1976, ApJ, 205, 762

Spitzer, L., \& Fitzpatrick, E. 1993, ApJ, 409, 299

Stark, A., Gammie, C., Wislon, R., et al. 1992, ApJS, 79, 77

Tull, R., MacQueen, P., Sneden, C., \& Lambert, D. 1995, PASP, 107, 251

Moos, H., Sembach, K., Vidal-Madjar, A., York, D., et al. 2002, ApJS, 140, 3

Morton, D. C. 1991, ApJS, 77, 119

Oegerle, W., Jenkins, E., Shelton, R., Bowen, D., \& Chayer, P. 2003, ApJ, submitted

Perrot, C., \& Grenier, I. 2003, A\&A, 404, 519

Redfield, S., \& Linsky, J. 2002, ApJS, 139, 439

Reynolds, R. 1984, ApJ, 282, 191

Richter, P, Sembach, K., Wakker, B., Savage, B., et al. 2001, ApJ, 559, 318

Vallerga, J. V., Vedder, P., Craig, N., \& Welsh, B. Y. 1993, ApJ, 411, 729

Van Steenberg, M., \& Shull, J. M. 1988, ApJS, 67, 225

Vennes, S., Theijll, P., Genova, G., \& Dupuis, J. 1997, ApJ, 480, 714

Verschuur, G. 1993, ApJ, 409, 205

Wakker, B. 2001, ApJS, 136, 463

Welsh, B. Y. Sfeir, D., Sirk, M., \& Lallement, R. 1999, A\&A, 352, 308

Welsh, B. Y., Sfeir, D., Sallmen, S., \& Lallement, R. 2001, A\&A, 372, 516

Ryans, R., Keenan, F., Sembach, K., \& Davies, R. 1997, MNRAS, Welsh, B. Y., Sallmen, S., Sfeir, D., Shelton, R., \& Lallement, R. 2002, 289, 83

Sahnow, D., Moos, H. W., Ake, T., et al. 2000, ApJ, 538, L7 A\&A, 394, 691 